\title{
Dynamic Sampling for Minimizing Crystal Damage Prior to Diffraction Data Collection
}

\author{
Garth J. Simpson \\ Professor of Chemistry, Department of Chemistry, Purdue University, West Lafayette, IN, 47907 \\ gsimpson@purdue.edu
}

Dynamic sampling, in which the preceding set of measurements informs the next sampling position, is shown to greatly reduce the total dose required for diffraction-based crystal positioning at synchrotron sources. The high speeds and sensitivities of photon counting array detectors enable automated crystal positioning from low-dose raster-scanned diffraction measurements, which are particularly useful for automated high throughput analyses, for analyses of small crystals, and for measurements in turbid media. However, even low doses can be shown to significantly impact the structures recovered through photoelectron-induced damage. These effects are exacerbated in room temperature diffraction measurements, both directly through the higher rate of x-ray induced crystal damage at elevated temperatures and indirectly through rapid diffusion of radicals produced in the solvent surrounding the crystals. Minimizing the total delivered X-ray dose required for reliable crystal centering has the potential to significantly improve the quality of the structures recovered, particularly for room temperature diffraction measurements. Toward this end, a supervised learning approach for dynamic sampling (SLADS) provided a factor of 3-10 fold reduction in dose required for crystal positioning based on low-dose diffraction scanning with no significant sacrifice in position confidence. We have demonstrated implementation of the SLADS acquisition algorithm at GM/CA at the Advanced Photon Source with a $5 \mu \mathrm{m}$ diameter beam. These proof-of-concept studies suggest SLADS can be implemented using existing hardware available at numerous existing synchrotron end stations, facilitating the possibility for broader implementation. Advantages and limitations of the approach will be critically evaluated.
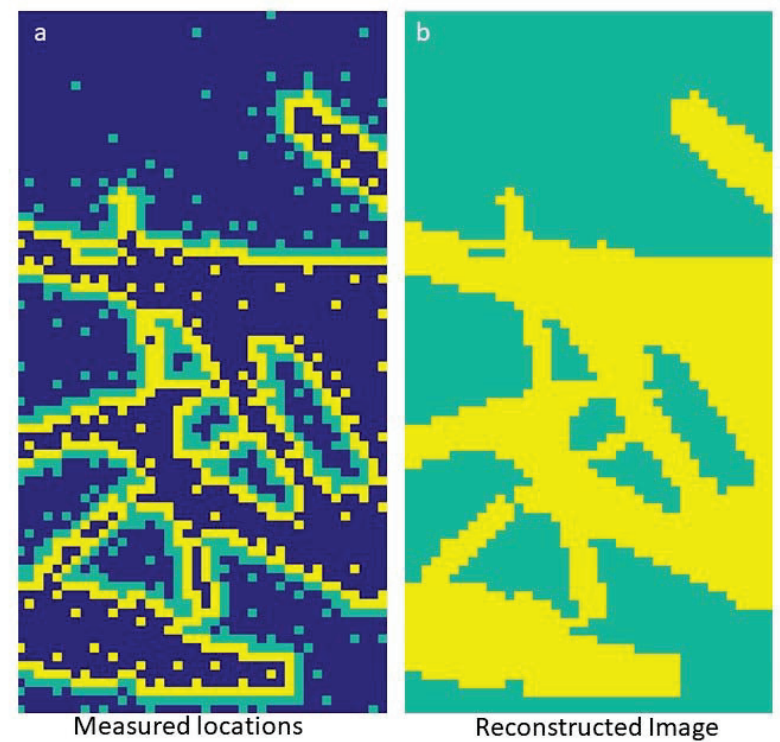

Reconstructed Image

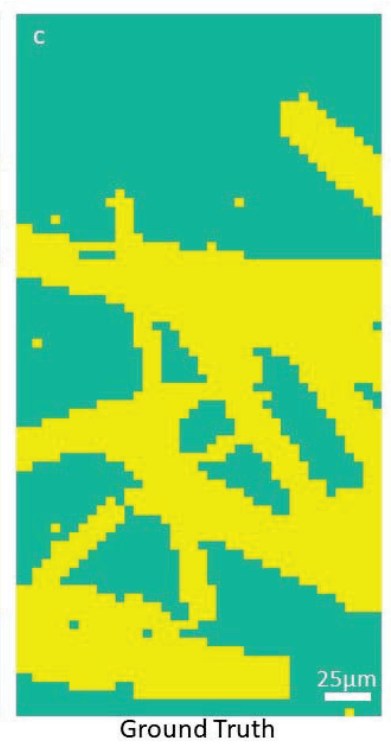

